\title{
PID and low-order controller design for guaranteed delay margin and pole placement
}

\author{
Hitay Özbay1® | A. Nazli Gündes²
}

${ }^{1}$ Dept of Electrical and Electronics Engineering, Bilkent University, Ankara, Turkey

${ }^{2}$ Dept of Electrical and Computer Engineering, University of California, Davis, Davis, California, USA

\section{Correspondence}

Hitay Özbay, Dept of Electrical and Electronics Engineering, Bilkent University, Ankara, Turkey. Email: hitay@bilkent.edu.tr

\begin{abstract}
This article provides a simple low-order controller design method (including PID controllers as special cases) for a class of unstable systems. First, PID controller design is considered for systems with two unstable poles and pole placement and delay margin issues are discussed. Then, a chain of integrators is considered with arbitrary stable dynamics in cascade. For a given desired minimum delay margin for this class of plants, a PID and low-order controller design method is obtained in terms of an inequality constraint on the sum of $k$ of the desired closed-loop poles, where $k$ is number of the integrators in the open-loop transfer function.
\end{abstract}

\section{K E Y W O R D S}

chain of integrators, PID control, time delay, unstable system

\section{1 | INTRODUCTION}

Low-order and PID controller design for unstable systems and systems with time delay is a challenging problem under various design specifications; see a relatively recent survey ${ }^{1}$ and also References 2-4.

In this article we will first consider plants with two unstable poles as in Reference 5, where PID tuning mechanisms are compared. For this class of systems explicit bounds for achievable delay margin have been studied in References 6,7 under PID and low-order controllers. Here we investigate closed-loop pole placement subject to a minimum for a lower bound of the delay margin. For this purpose we use a computationally attractive lower bound of the delay margin, determined from a weighted $\mathscr{H}_{\infty}$-norm of the complementary sensitivity function, as in References 8-10.

Next we consider a chain of $k$ integrators in cascade with arbitrary stable transfer function (i.e., in this case the plant order can be arbitrarily large but all the unstable poles are at the origin). Again, for a minimum of the delay margin lower bound, PID and low-order controllers are designed to achieve partial pole placement. It will be shown that the controller is determined from $k$ free parameters $(k+1$ if an integral action is desired in the controller). Also, the relation between these parameters and the closed-loop pole locations will be discussed. We should indicate that this class of plants is also studied widely in the literature, see, for example, References 11,12, where the plant is stabilized by multiple delayed feedback controllers and application examples are given in References 13,14. For pole placement issues for delay systems see a recent article ${ }^{15}$ and its references.

The article is organized as follows. In the next section the problems studied are formulated. In Section 3, PID controller design for plants with two unstable poles is discussed. In Section 4, PID and low-order controllers are designed for a chain of $k$ integrators subject to a minimum of the lower bound of the delay margin. Examples are given in Section 5 and concluding remarks are made in Section 6. 


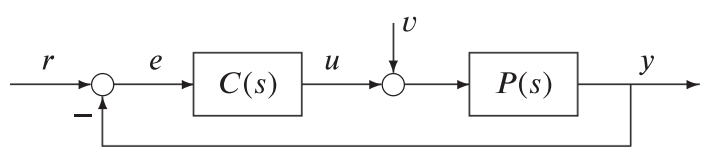

F I G U R E 1 Feedback control system $(C, P)$

Notation: Let $\mathbb{C}$ denote complex numbers. The closed right-half-plane (RHP) is $\mathbb{C}_{+}=\{s \in \mathbb{C} \mid \mathfrak{R} e(s) \geq 0\}$, and the open left-half-plane (LHP) is $\mathbb{C}_{-}=\{s \in \mathbb{C} \mid \mathfrak{R} e(s)<0\}$. The region of instability is the extended closed RHP, that is, $\mathbb{C}_{+e}=$ $\mathbb{C}_{+} \cup\{\infty\}$. Real and positive real numbers are denoted by $\mathbb{R}$ and $\mathbb{R}_{+}$, respectively; $\mathcal{R}_{p}$ and $\mathcal{R}_{\infty}$ denote real proper and strictly proper rational functions of $s ; S \subset \mathcal{R}_{p}$ is the stable subset with no poles in $\mathbb{C}_{+e}$. The space $\mathscr{H}_{\infty}$ is the set of all bounded analytic functions in $\mathbb{C}_{+} . f \in \mathscr{H}_{\infty}$, the norm $\|\cdot\|$ is defined as $\|f\|:=\operatorname{ess~sup}_{s \in \mathbb{C}_{+}}|f(s)|$, where ess sup denotes the essential supremum. The degree of the polynomial $d$ is denoted by $\operatorname{deg}(d)$. For simplicity, we drop $(s)$ in transfer functions such as $P(s)$.

\section{2 | PROBLEM FORMULATION}

Consider the standard feedback system formed by controller $C$ and plant $P$, denoted by $(C, P)$, shown in Figure 1 .

The feedback system $(C, P)$ is stable if transfer functions from $r$ and $v$ to $u$ and $y$ are in $\mathscr{H}_{\infty}$ and this is equivalent to having $S=(1+P C)^{-1}, T=1-S, C S$ and $P S$ in $\mathscr{H}_{\infty}$. Assuming that there is no unstable pole-zero cancelation in the product $P(s) C(s)$, the feedback system is stable if and only if the all the poles of $T$ are in $\mathbb{C}_{-}$. In this article low-order and PID controllers are designed for various type of unstable plants. The delay margin (DM) of a stable feedback system $(C, P)$ is the largest $\tau_{\max }$ such that $\left(C, e^{-\tau s} P\right)$ is stable for all $\tau \in\left[0, \tau_{\max }\right)$.

The main problem studied in the article can be stated as follows: given an unstable $P \in \mathcal{R}_{p}$, design a low-order (or PID type) controller such that

(i) (pole placement): the dominant closed-loop system poles are at the desired locations in $\mathbb{C}_{-}$, and

(ii) the delay margin is larger than a specified value $h$.

In general, explicit closed-form expression for the delay margin is not available; but it can be computed numerically from the gain crossover frequency and the phase margin. A lower bound of the delay margin is determined as follows: consider a stable feedback system $(C, P)$ and examine $S_{h}:=\left(1+e^{-h s} P C\right)^{-1}$

$$
S_{h}=\left(1+P C+\left(e^{-h s}-1\right) P C\right)^{-1}=S\left(1+\left(e^{-h s}-1\right) T\right)^{-1} .
$$

Note that

$$
\left\|\frac{e^{-h s}-1}{h s}\right\|=1 .
$$

Therefore, the delayed feedback system $\left(C, e^{-h s} P\right)$ is stable if

$$
\|s T\|<\frac{1}{h} .
$$

Hence, the DM of $(C, P)$ is at least $\|s T\|^{-1}$. This fact will be used in the next sections to take care of the second design condition (ii).

\section{3 | SECOND-ORDER UNSTABLE PLANTS}

Consider a second-order unstable plant $P \in \mathcal{R}_{p}$

$$
P(s)=\frac{1}{\left(s-p_{1}\right)\left(s-p_{2}\right)}, \quad \operatorname{Re}\left(p_{i}\right)>0, \quad i=1,2 .
$$


In recent works ${ }^{6,7}$ delay margin optimizing controllers have been investigated for this class of plants. It has been shown that largest possible delay margin is obtained by using PD controllers, and adding a "small" integral action leads to a "small" reduction in the DM. On the other hand, it is well known that maximizing the DM typically leads to slow step response and may have other undesirable closed-loop system characteristics, for example, small gain margin. Nevertheless, it is always good to know what the largest achievable DM is. In this section, we design PID controllers for a guaranteed DM with a closed-loop pole placement objective. Note that (4) includes two real poles and two complex conjugate poles cases. The discussion below captures both cases.

For the plant (4) we will design a PID controller in the form

$$
C_{P I D}(s)=K_{P}+\frac{K_{I}}{s}+K_{D} s=K_{D} \frac{\left(s-z_{1}\right)\left(s-z_{2}\right)}{s},
$$

where $K_{P}, K_{I}, K_{D}$ are nonnegative and

$$
z_{1,2}=-\frac{K_{P}}{2 K_{D}}\left(1 \pm \sqrt{1-4 \frac{K_{I} K_{D}}{K_{P}^{2}}}\right)
$$

If $0<K_{I} K_{D} / K_{P}^{2} \leq 1 / 4$, then the zeros are real and $z_{2} \leq z_{1}<0$. In this case, typical root locus is as shown in Figure 2 , where $\left\{-\beta_{1},-\beta_{2},-\beta_{3}\right\}$ are the set of closed-loop system poles. For the case $K_{I} K_{D} / K_{P}^{2}>1 / 4$ we have complex conjugate zeros; typical root locus for this case is as shown in Figure 3.

One of the closed-loop pole placement objectives we consider here is to choose $K_{P}, K_{I}$ and $K_{D}$ such that closed-loop poles are real, as shown in Figures 2 and 3,

$$
-\beta_{3} \leq-\beta_{2} \leq-\beta_{1}<0 .
$$

Before we investigate further constraints in the design, let us discuss the lower bound of the delay margin determined from (3). The complementary sensitivity function is

$$
T(s)=K_{D} \frac{\left(s-z_{1}\right)\left(s-z_{2}\right)}{\left(s+\beta_{1}\right)\left(s+\beta_{2}\right)\left(s+\beta_{3}\right)}
$$

so,

$$
\|s T\|=K_{D}\left\|\frac{s}{\left(s+\beta_{1}\right)} \frac{\left(s-z_{1}\right)}{\left(s+\beta_{2}\right)} \frac{\left(s-z_{2}\right)}{\left(s+\beta_{3}\right)}\right\| .
$$

In the real zeros case, from Figure 2, it is clear that

$$
\|s T\| \leq K_{D} \quad \text { because } \quad\left\|\frac{s-z_{i-1}}{s+\beta_{i}}\right\| \leq 1, \quad i=1,2,3,
$$

where we used the notation $z_{0}=0$. For the design method proposed below we will see that (10) holds even if the zeros are complex conjugate because we will have $\left|z_{i}\right| \leq \beta_{2}, i=1,2$, (this will be formally proven at the end of the section).

F I G U R E 2 Typical root locus for real zeros [Colour figure can be viewed at wileyonlinelibrary.com]

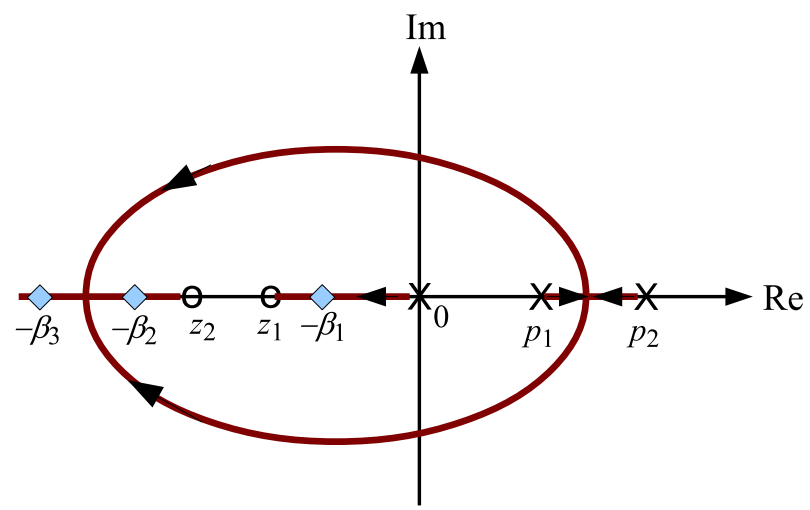




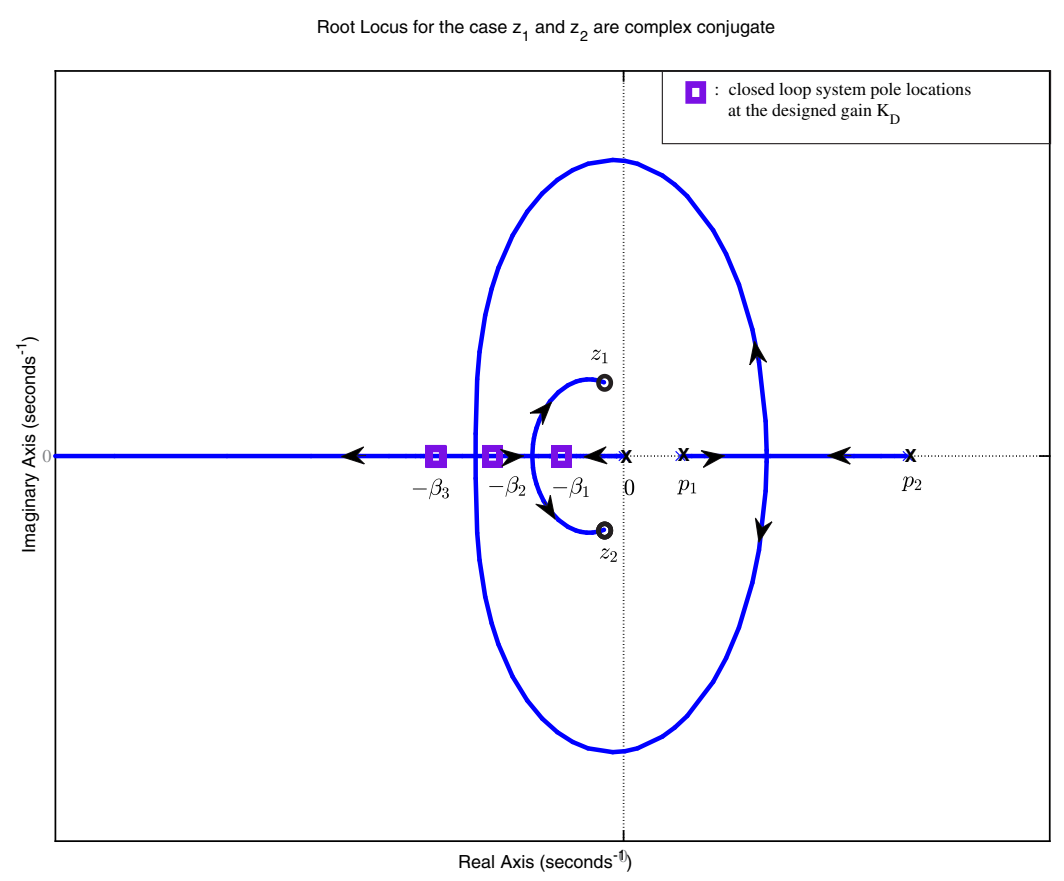

F I G U R E 3 Typical root locus for complex conjugate zeros [Colour figure can be viewed at wileyonlinelibrary.com]

Hence, it can be concluded that if $h$ (a minimum acceptable delay margin) is given, then $K_{D}$ should be chosen to satisfy

$$
K_{D}<\frac{1}{h}
$$

Now we can further discuss the implications of the bound (11) on the pole placement, that is, the selection of $z_{1}, z_{2}$ (equivalently $K_{P}$ and $K_{I}$ ) and their relations with $\beta_{1}, \beta_{2}, \beta_{3}$. The characteristic equation of the feedback system $(C, P)$ is

$$
s^{3}+\left(K_{D}-\left(p_{1}+p_{2}\right)\right) s^{2}+\left(p_{1} p_{2}+K_{P}\right) s+K_{I}=0
$$

and it is desired to be equal to

$$
\left(s+\beta_{1}\right)\left(s+\beta_{2}\right)\left(s+\beta_{3}\right)=s^{3}+\left(\beta_{1}+\beta_{2}+\beta_{3}\right) s^{2}+\left(\beta_{1} \beta_{2}+\beta_{1} \beta_{3}+\beta_{2} \beta_{3}\right) s+\beta_{1} \beta_{2} \beta_{3}=0 .
$$

From the above identity we have

$$
K_{D}=\left(p_{1}+p_{2}\right)+\left(\beta_{1}+\beta_{2}+\beta_{3}\right)
$$

Thus, as $h$ increases in (11) the largest allowable value of the sum $\left(\beta_{1}+\beta_{2}+\beta_{3}\right)$ decreases. How should we select controller parameters so that $\left(\beta_{1}+\beta_{2}+\beta_{3}\right)$ is small? From the root locus figures it is clear that choosing $\beta_{1}=\beta_{0}$ (a given acceptable value) and $\beta_{2}=\beta_{3}=\beta$ leads to a good design from the pole placement and DM perspectives. What is the smallest possible $\beta$ in terms of $\beta_{0}$ ? What is the resulting DM lower bound? The answers are given by the following result and its proof.

Proposition 1. Consider the feedback system with plant (4) and controller (5). Let $-\beta_{3}=-\beta_{2}=-\beta<-\beta_{1}=\beta_{0}<0$ denote the desired closed-loop system pole locations. A lower bound of the largest achievable delay margin is

$$
h_{\max }^{o}=\frac{1}{\left(p_{1}+p_{2}\right)+\sqrt{3 p_{1} p_{2}}} .
$$

Proof. From the coefficients of $s^{1}$ and $s^{0}$ terms in the characteristic equation we see that

$$
\beta=\sqrt{\beta_{o}^{2}+K_{P}+p_{1} p_{2}}-\beta_{o}
$$


and hence

$$
\frac{1}{h}=p_{1}+p_{2}+\beta_{o}+2 \beta=p_{1}+p_{2}+2 \sqrt{\beta_{o}^{2}+K_{P}+p_{1} p_{2}}-\beta_{o}, \quad K_{P}=\frac{\left(\beta_{o}-\left(p_{1}+p_{2}\right)+1 / h\right)^{2}}{4}-\left(\beta_{o}^{2}+p_{1} p_{2}\right) .
$$

Moreover, $K_{I}=\beta_{o} \beta^{2}$. Since $K_{P}>0$,

$$
h<\left(\left(p_{1}+p_{2}\right)+2 \sqrt{\beta_{o}^{2}+p_{1} p_{2}}-\beta_{o}\right)^{-1}=: h_{\max }\left(\beta_{0}\right) .
$$

For relatively large values of $\beta_{o}$ the inequality (17) shows an inverse relation between $\beta_{o}$ and $h_{\max }$, maximum of the lower bound of the DM considered here. For fast step response and small steady-state error for unit ramp reference input we need a large value for $\beta_{o}$, on the other hand this will make the admissible values of $h$ small. The relation (17) gives an optimal value of $\beta_{o}$ which maximizes $h_{\max }$ :

$$
h_{\max }^{o}=\frac{1}{\left(p_{1}+p_{2}\right)+\sqrt{3 p_{1} p_{2}}} \text { is obtained for } \beta_{o}^{o}=\sqrt{\frac{p_{1} p_{2}}{3}} .
$$

Figure 4 shows $h_{\max }$ versus $\beta_{o}$ for $p_{1}=0.2$ and a few values of $p_{2}$.

Thus the PID design procedure can now be summarized as follows: given $p_{1}$ and $p_{2}$ choose

$$
\beta_{0}=\beta_{o}^{o}=\sqrt{p_{1} p_{2} / 3}
$$

and pick an acceptable level of DM, $h$, subject to

$$
h<h_{\max }^{o}=\frac{1}{\left(p_{1}+p_{2}\right)+\sqrt{3 p_{1} p_{2}}} .
$$

Let

$$
K_{P}=0.25\left(\sqrt{p_{1} p_{2} / 3}+h^{-1}-\left(p_{1}+p_{2}\right)\right)^{2}-4 p_{1} p_{2} / 3
$$

and define

$$
\beta=\sqrt{K_{P}+4 p_{1} p_{2} / 3}-\sqrt{p_{1} p_{2} / 3} .
$$

F I G U R E $4 \quad h_{\max }$ versus $\beta_{o}$ [Colour figure can be viewed at wileyonlinelibrary.com]

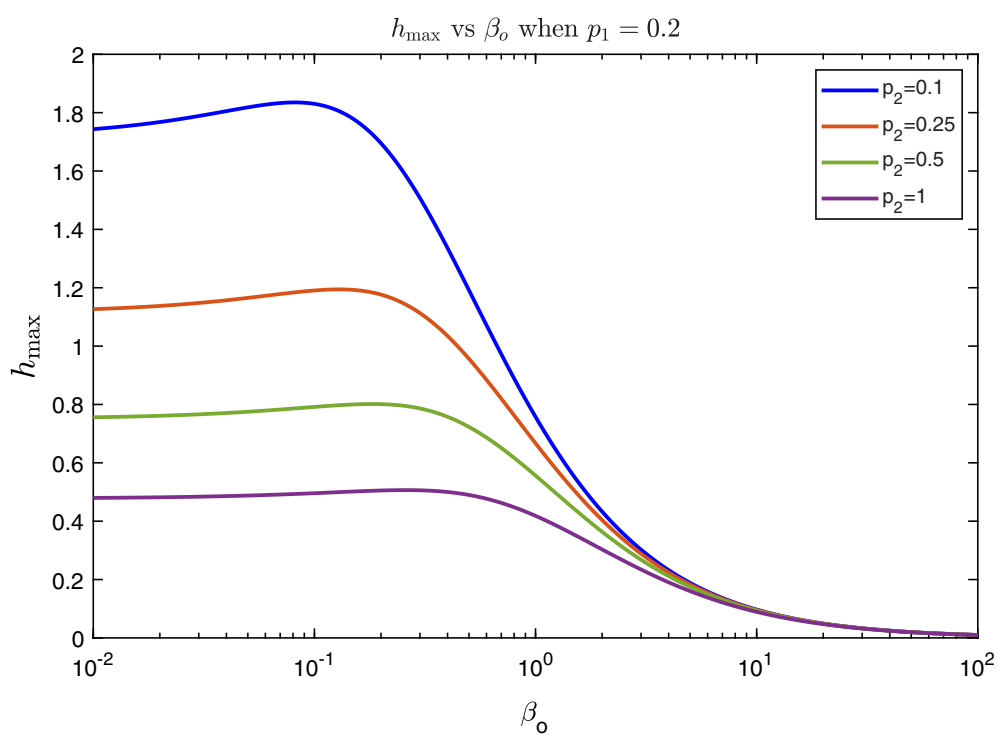


Then, integral and derivative gains are computed as

$$
K_{D}=1 / h, \quad K_{I}=\beta_{o} \beta^{2} .
$$

Remark 1. For all $h<h_{\max }^{o}$ the resulting $K_{P}$ in (21) is positive, and in this case $\beta$ defined in (22) is greater than $\beta_{0}$ as desired. Now it is left to prove the claim made earlier, that $\left|z_{i}\right|<\beta, i=1,2$, in this design when $z_{1}$ and $z_{2}$ are complex conjugate. To see this fact, recall that

$$
\left|z_{i}\right|^{2}=K_{I} / K_{D}=h \beta_{o} \beta^{2}
$$

Therefore, $\left|z_{i}\right|=\beta \sqrt{h \beta_{0}}$, with $\beta_{o}$ is defined as (19), and $h$ satisfying the inequality (20). Furthermore,

$$
h \beta_{o}<h_{\max }^{o} \beta_{o}=\frac{\sqrt{p_{1} p_{2} / 3}}{\left(p_{1}+p_{2}\right)+\sqrt{3 p_{1} p_{2}}}<1,
$$

which proves the claim.

\section{4 | PLANTS WITH A CHAIN OF INTEGRATORS}

In the previous section we considered second-order unstable plants. In this section we consider arbitrary-order plants with $k$ poles at $s=0$, but no other poles in $\mathbb{C}_{+}$. As mentioned in the Introduction, this is an interesting class of plants in various applications, including high precision position control of multi-link robot arm.

The plant is in the form

$$
P(s)=\frac{1}{s^{k}} G_{k}(s),
$$

where $k \geq 1, G_{k}(0) \neq 0$ and $G_{k}$ has no poles in $\mathbb{C}_{+}$. In other words, the plant has exactly $k$ poles at $s=0$ and these are the only unstable poles. Note that $G_{k}$ may be improper, but it is possible to make it proper by a cascade connection with a strictly proper stable minimum phase transfer function. The plant may have any number of $\mathbb{C}_{-}$poles and there are no restrictions on the plant zeros. Moreover, the plant may be infinite dimensional and in particular it may contain time delays (input-output delay, as well as state delays internally).

Lemma 1. Let $\beta_{i} \in \mathbb{R}_{+}, i=1, \ldots, k$, be nonnegative real constants. Define the polynomial $\phi_{k}$ as:

$$
\phi_{k}(s):=\prod_{i=1}^{k}\left(s+\beta_{i}\right)
$$

Then the following norm equality holds:

$$
\left\|S\left(1-\frac{s^{k}}{\phi_{k}(s)}\right)\right\|=\sum_{i=1}^{k} \beta_{i} .
$$

Proof. This is a special case of lemma 3.1 of Reference 10.

The following result gives various types of low-order controllers (including PID) stabilizing $e^{-h s} P(s)$ for a given lower bound of the DM, $h>0$.

Proposition 2. Let $P$ be given in (26) with $G_{k}(0) \neq 0$ and $G_{k}$ has no poles in $\mathbb{C}_{+}$. Choose any $Q \in \mathscr{H}_{\infty}$ that satisfies $Q(0)=G_{k}(0)^{-1}, s^{k-1} Q \in \mathscr{H}_{\infty}$, and $G_{k} Q \in \mathscr{H}_{\infty}$ (note that $Q$ may have to be strictly proper). Define the polynomials $\phi_{k}, \phi_{(k+1)}$ as:

$$
\phi_{k}(s):=\prod_{i=1}^{k}\left(s+\beta_{i}\right), \quad \phi_{(k+1)}(s):=\prod_{i=1}^{(k+1)}\left(s+\beta_{i}\right)
$$

Define $B \in \mathbb{R}_{+}$as

$$
B:=\left\|\frac{G_{k} Q-1}{s}\right\|+h\left\|G_{k} Q\right\| .
$$


(a) Strong stabilization (no integral action in the controller): For $i=1, \ldots, k$, let $\beta_{i} \in \mathbb{R}_{+}$satisfy

$$
\sum_{i=1}^{k} \beta_{i}<\frac{1}{B}
$$

Then the controller $C_{s} \in \mathscr{H}_{\infty}$ given in (32) stabilizes the delayed plant $e^{-h s} P$ :

$$
C_{s}(s)=\left(\phi_{k}(s)-s^{k}\right) Q(s)
$$

(b) Integral-action stabilization: For $i=1, \ldots,(k+1)$, let $\beta_{i} \in \mathbb{R}_{+}$satisfy

$$
\sum_{i=1}^{(k+1)} \beta_{i}<\frac{1}{B}
$$

Then the integral-action controller $C_{i}$ given in (34) stabilizes the delayed plant $e^{-h s} P$ :

$$
C_{i}(s)=\left(\frac{\phi_{(k+1)}(s)-s^{(k+1)}}{s}\right) Q(s)=C_{s}(s)+\frac{\beta_{(k+1)} \phi_{k}(s)}{s} Q(s) .
$$

Proof. Write $P$ as

$$
P(s)=\left(\frac{s^{k}}{\phi_{k}(s)}\right)^{-1}\left(\frac{s^{k}}{\phi_{k}(s)} P(s)\right) .
$$

(a) Let $M_{k}$ be defined as

$$
M_{k}:=\frac{s^{k}}{\phi_{k}}+C_{s} e^{-h s} \frac{s^{k}}{\phi_{k}} P=\frac{s^{k}}{\phi_{k}}+\frac{\left(\phi_{k}-s^{k}\right)}{\phi_{k}} e^{-h s} G_{k} Q=1+\frac{s\left(\phi_{k}-s^{k}\right)}{\phi_{k}}\left(\frac{G_{k} Q-1}{s}+\frac{\left(e^{-h s}-1\right)}{h s} h G_{k} Q\right) .
$$

Since

$$
\left\|\frac{e^{-h s}-1}{h s}\right\|=1
$$

using the norm equality (28) in Lemma 1, if the small gain condition (31) holds, it follows that

$$
\begin{aligned}
\left\|\frac{s\left(\phi_{k}-s^{k}\right)}{\phi_{k}}\left(\frac{G_{k} Q-1}{s}+\frac{\left(e^{-h s}-1\right)}{h s} h G_{k} Q\right)\right\| & \leq\left\|\frac{s\left(\phi_{k}-s^{k}\right)}{\phi_{k}}\right\|\left(\left\|\frac{G_{k} Q-1}{s}\right\|+\left\|\frac{e^{-h s}-1}{h s}\right\|\left\|h G_{k} Q\right\|\right) \\
& \leq \sum_{i=1}^{k} \beta_{i}\left(\left\|\frac{G_{k} Q-1}{s}\right\|+h\left\|G_{k} Q\right\|\right)<1 .
\end{aligned}
$$

Therefore, $M_{k}$ is a unit, and hence, $C_{k}$ is a stabilizing controller for $e^{-h s} P$.

(b) Write the integral-action controller in (34) as

$$
C_{i}=\left(\frac{s}{\left(s+\beta_{(k+1)}\right)}\right)^{-1}\left(\frac{\left(\phi_{(k+1)}-s^{(k+1)}\right)}{\left(s+\beta_{(k+1)}\right)} Q\right) .
$$

Let $M_{k i}$ be defined as

$$
M_{k i}:=\frac{s^{(k+1)}}{\phi_{(k+1)}}+\frac{\left(\phi_{(k+1)}-s^{(k+1)}\right)}{\phi_{(k+1)}} e^{-h s} G_{k} Q=1+\frac{\left(\phi_{(k+1)}-s^{(k+1)}\right) s}{\phi_{(k+1)}}\left(\frac{G_{k} Q-1}{s}+\frac{\left(e^{-h s}-1\right)}{h s} h G_{k} Q\right) .
$$

If the small gain condition (33) holds, then using the norm equality (28) in Lemma 1, it follows that $M_{k i}$ is a unit. 
Remark 2 (Closed-loop system poles). The above analysis shows that in case (a), the closed-loop system poles are the zeros of the unit $M_{k}(s)$. If $Q \in \mathscr{H}_{\infty}$ is selected in such a way that $B$, defined by (30), is much smaller than 1, then $M_{k}$ is close to 1 , which means that the dominant closed-loop system poles are near $-\beta_{1}, \ldots,-\beta_{k}$. Similarly in case (b), dominant closed-loop system poles are near $-\beta_{1}, \ldots,-\beta_{(k+1)}$, when $B \ll 1$, which may be possible to achieve only when $h$ is small.

Remark 3 (The relation between $\beta_{i}$ 's and $h$ ). Recall that for various control objectives we would like to make $\beta_{i}$ 's as large as possible. On the other hand, they are restricted by $\sum \beta_{i}<1 / B$, where $B$ is defined in (30). It is clear that there is an inverse relationship between $1 / B$ and $h$. For a fixed $h$, to make $1 / B$ large, we need to select $Q(s)$ in such a way that $\left\|G_{k} Q\right\|$ and $\left\|R_{Q}\right\|$ are small, where

$$
R_{Q}(s):=\frac{G_{k}(s) Q(s)-1}{s}
$$

Since $Q(0)=1 / G_{k}(0)$, it is impossible to have $\left\|G_{k} Q\right\|<1$; but with a judicious choice it may be possible to get $\left\|G_{k} Q\right\|=1$. Then, among such possibilities we need to pick the one which minimizes $\left\|R_{Q}\right\|$, to get the largest possible $1 / B$. In general, $Q$ should be chosen to make $B=\left\|R_{Q}\right\|+h\left\|G_{k} Q\right\|$ small for a given fixed $h$.

Remark 4 (Controller order). The order of the controllers in Proposition 2 depends on the choice of the free parameter $Q \in \mathscr{H}_{\infty}$. If no integral-action required, then the order of the controller $C_{s}$ in (32) can be as low as $(k-1)$ (one less than the number of plant poles at $s=0$ ). With integral-action, the order of the controller $C_{i}$ in (34) can be as low as $k$ (same as the number of plant poles at $s=0$ ).

Corollary 1 below states a special case for strictly proper plants, where the parameter $Q$ can be chosen so that $C_{s}$ in (32) becomes a (proper) PD controller, and $C_{i}$ in (34) becomes a PID controller of the form

$$
C_{P D}=K_{p}+\frac{K_{d} S}{\tau_{d} S+1}, \quad C_{P I D}=C_{P D}+\frac{K_{i}}{s} .
$$

The pole at $-1 / \tau_{d}$ is included for implementation, where $\tau_{d}>0$ is typically very small so that the second term in $C_{P D}$ acts like a derivative.

Corollary 1 ( $P D$ and PID controllers for $k=1$ ). In addition to the assumptions of Proposition 2, suppose that $P$ is strictly proper. Let $k=1$, that is, $G_{k}(s)=s P(s)$.

(a) (PD controller): Choose any $\tau_{d} \in \mathbb{R}_{+}$and any $K \in \mathbb{R}$. In (32), let

$$
Q(s)=G_{k}(0)^{-1}+\frac{K s}{\tau_{d} s+1} .
$$

Define the norm B as

$$
B:=\left\|\frac{G_{k} G_{k}(0)^{-1}-1}{s}+G_{k} \frac{K}{\tau_{d} s+1}\right\|+h\left\|G_{k} Q\right\| .
$$

For $\beta_{1} \in \mathbb{R}_{+}$satisfying $\beta_{1}<B^{-1}$, the PD controller $C_{S}=C_{P D} \in S$ given in (44), derived from (32), stabilizes the delayed plant $e^{-h s} P$ :

$$
C_{P D}=\beta_{1}\left[G_{k}(0)^{-1}+\frac{K S}{\tau_{d} S+1}\right]
$$

For $K=0$, the controller in (44) is a proportional controller.

(b) (PID controller): Let $Q \in S$ be as in (42). With B defined as (43), for $\beta_{1}, \beta_{2} \in \mathbb{R}_{+}$satisfying $\left(\beta_{1}+\beta_{2}\right)<B^{-1}$ the cascade $P I-P D$ controller $C_{P I D}$ given in (45), derived from (34), stabilizes the delayed plant $e^{-h s} P$ :

$$
C_{P I D}=\left[\left(\beta_{1}+\beta_{2}\right)+\frac{\beta_{1} \beta_{2}}{s}\right]\left[G_{k}(0)^{-1}+\frac{K s}{\tau_{d} s+1}\right] .
$$

For $K=0$, the controller in (45) is a proportional + integral controller.

For plants that have two poles at $s=0$, the lowest order of stabilizing controllers designed based on Proposition 2 is one. However, depending on $G_{k}$, the free parameter $Q \in \mathscr{H}_{\infty}$ may have to be strictly proper In this case, the controllers in 
(32) and (34) cannot be in PD and PID forms. Corollary 2 below derives a first-order controller for strictly proper plants, where the parameter $Q$ can be chosen so that $C_{S}$ in (32) becomes a (proper) PD controller, and $C_{i}$ in (34) becomes a PID controller.

Corollary 2 (Low-order controllers for $k=2$ ). In addition to the assumptions of Proposition 2, suppose that $P$ is strictly proper. Let $k=2$, that is, $G_{k}(s)=s^{2} P(s)$.

(a) Choose any $\tau_{d} \in \mathbb{R}_{+}$and any $K \in \mathbb{R}$. In (32), for any $\alpha \in \mathbb{R}_{+}$, let

$$
Q(s)=\frac{1}{\alpha s+1}\left(G_{k}(0)^{-1}+\frac{K s}{\tau_{d} s+1}\right) .
$$

With $Q$ as in (46), for $\beta_{1}, \beta_{2} \in \mathbb{R}_{+}$satisfying the inequality (31), the controller $C_{S} \in S$ given in (47), derived from (32), stabilizes the delayed plant $e^{-h s} P$ :

$$
C_{s}(s)=\left[\frac{\left(\beta_{1}+\beta_{2}\right) s+\beta_{1} \beta_{2}}{\alpha s+1}\right]\left[G_{k}(0)^{-1}+\frac{K s}{\tau_{d} s+1}\right]
$$

For $K=0$, the controller in (47) is a first-order controller.

(b) With $Q$ as in (46), for $\beta_{1}, \beta_{2}, \beta_{3} \in \mathbb{R}_{+}$satisfying the inequality (33), the controller $C_{i}$ given in (48), derived from (34), stabilizes the delayed plant $e^{-h s} P$ :

$$
C_{i}(s)=\left[\left(\left(\beta_{1}+\beta_{2}\right) s+\beta_{1} \beta_{2}\right)\left(1+\frac{\beta_{3}}{s}\right)+\beta_{3}\right] Q(s) .
$$

\section{5 | EXAMPLES}

\section{1 | Plants with two unstable poles}

Example 1 (Two real poles). Consider the plant (4) with $p_{1}=0.2$ and $p_{2}=1$. Applying the design procedure proposed in Section 2, we see that $\beta_{o}^{o}=\sqrt{p_{1} p_{2} / 3}=0.2582$ and this leads to $h_{\max }^{o}=0.5064 \mathrm{~s}$. Now if we select $h=0.4 \mathrm{~s}$, we should set $K_{D}=1 / h=2.5$. Furthermore, from (21) we have $K_{P}=0.3404$ and this leads to $\beta=0.5209$, which gives $K_{I}=\beta_{0}^{o} \beta^{2}=0.0701$. Hence,

$$
C_{P I D}(s)=0.3404+\frac{0.0701}{s}+2.5 \mathrm{~s}
$$

The feedback system has one pole at -0.2582 and double pole at -0.5209 , with the actual resulting delay margin of $0.4439 \mathrm{~s}$. The step responses of the feedback system under different values of the delay are shown in Figure 5.

F I G U R E 5 Step response of the feedback $\operatorname{system}\left(p_{1}=0.2, p_{2}=1.0\right)$ [Colour figure can be viewed at wileyonlinelibrary.com]

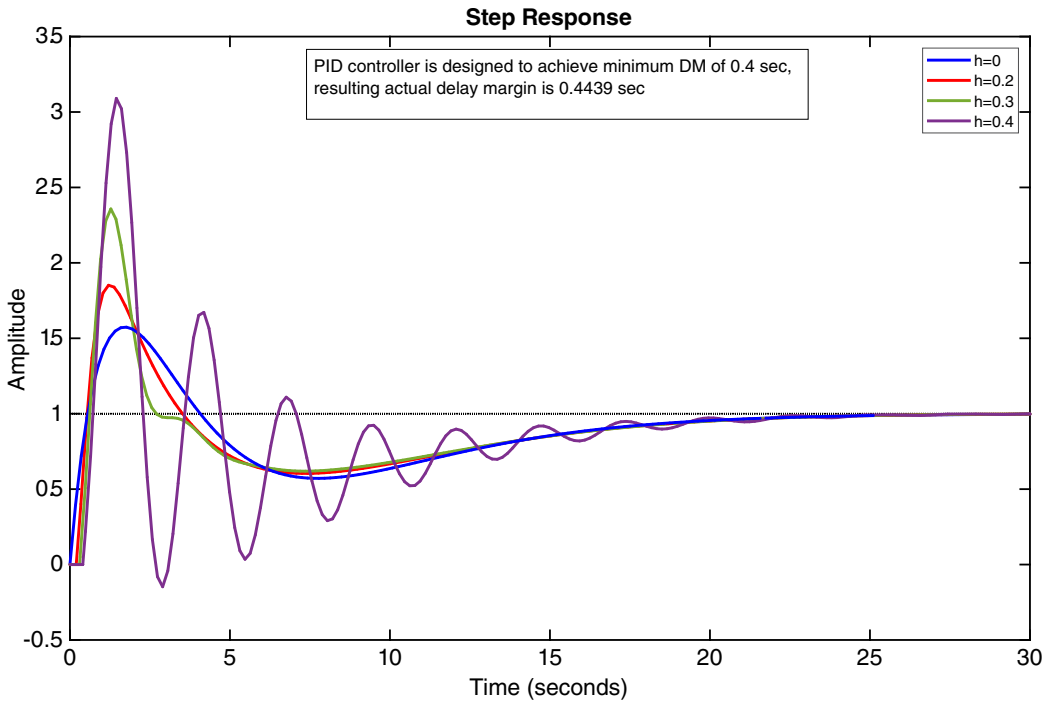




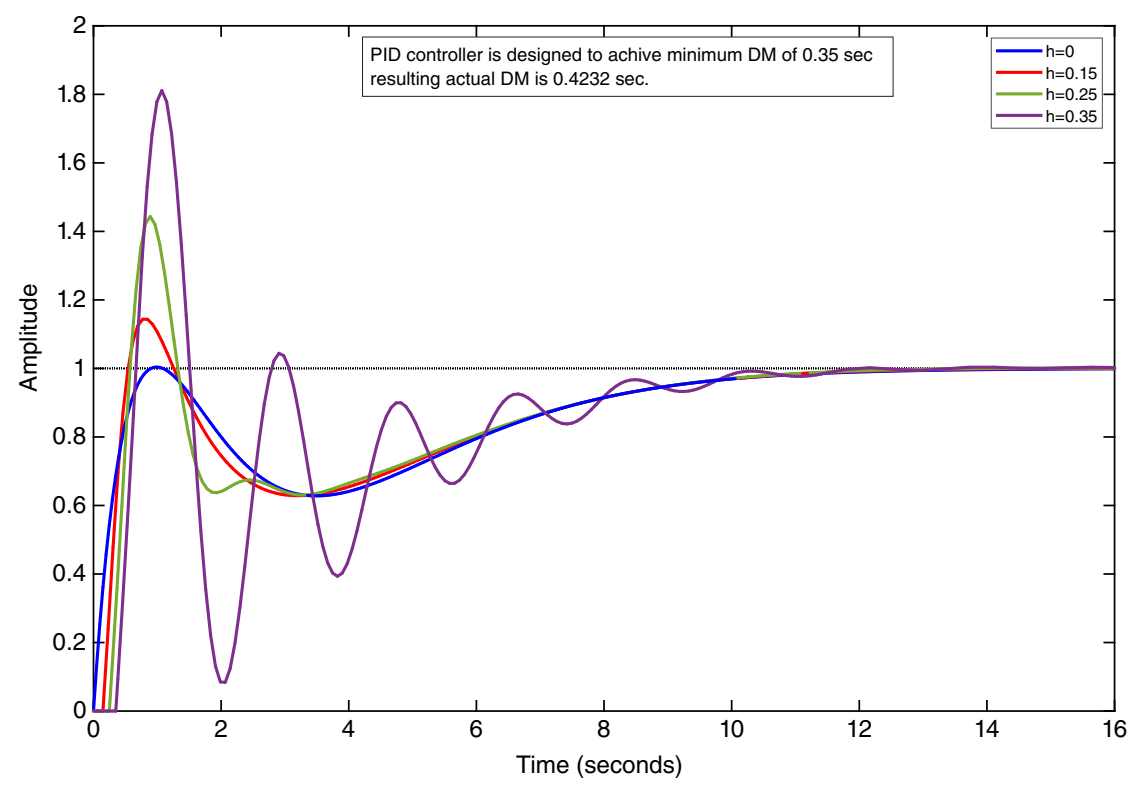

F I G U R E 6 Step response of the feedback system $\left(p_{1,2}=0.2 \pm j\right)$ [Colour figure can be viewed at wileyonlinelibrary.com]

Example 2 (Two complex conjugate poles). Now let the plant (4) be defined by the complex conjugate poles $p_{1,2}=0.2 \pm j 1$. The procedure of Section 2, leads to $\beta_{o}^{o}=\sqrt{p_{1} p_{2} / 3}=0.5888$ which gives $h_{\max }^{o}=0.4616 \mathrm{~s}$. Accordingly, if we select $h=0.35 \mathrm{~s}$ as the minimum allowable DM, then $K_{D}=1 / h=2.8571$. As before, from (21) we have $K_{P}=0.9328$ and this leads to $\beta=0.9342$, which gives $K_{I}=\beta_{0}^{o} \beta^{2}=0.5138$. Therefore,

$$
C_{P I D}(s)=0.9328+\frac{0.5138}{s}+2.8571 \mathrm{~s}
$$

The feedback system has one pole at -0.5888 and double pole at -0.9342 , with the actual resulting delay margin of $0.4232 \mathrm{~s}$. The step responses of the feedback system under different values of the delay are shown in Figure 6.

\section{2 | Plants with a chain of integrators}

Example 3 (Fourth-order plant with one pole at $s=0$ ). Consider the plant

$$
P=\frac{s-1}{s(s+6)\left(s^{2}+8 s+17\right)}
$$

where $G_{k}=(s P)$. Let $Q=G_{k}(0)^{-1}=-102$. Then $B \in \mathbb{R}_{+}$in $(30)$ is computed as

$$
\left\|\frac{G_{k} Q-1}{S}\right\|=1.6373, \quad\left\|G_{k} Q\right\|=1.901, \quad B=1.6373+h 1.901 .
$$

For $h=1 \mathrm{~s}$, a constant controller $C_{s}$ is obtained from (32) as $C_{s}=-102 \beta_{1}$ for $\beta_{1}<B^{-1}=0.2826$ satisfying (31). For an integral-action controller, choosing $\beta_{1}=\beta_{2}=0.141$ satisfying (33), a PI controller is obtained from (34) as

$$
C_{i}=-102 \frac{(0.282 s+0.0199)}{s}
$$

With the PI controller in (53), the system has a delay margin of $D M=2.8503 \mathrm{~s}$. The step responses for $h=0,0.5,0.95,1.75$ are shown in Figure 7. The response is slow as expected since $Q(s)$ is chosen for a low-order $C(s)$ (a PI controller). Other $Q$ choices may give faster response. Since $P$ has RHP zero and relative degree 3, performance degradation with delay is not surprising. 
Let us now discuss the design of $C_{P I D}$ defined by (45). In this case we have a free parameter, $K$ (the derivative action gain, when $\tau_{d} \rightarrow 0$ ) to choose. We may now ask: what is the optimal choice of $K$ which makes $B$, as small as possible, for a fixed $h$ ? This is quite relevant for maximizing the allowable sum of $\beta_{i}$ 's. Recall that $B$, (43), consists of two terms, $B=B_{1}+B_{2}$, where

$$
B_{1}:=\left\|\frac{G_{k}(s) G_{k}(0)^{-1}-1}{s}+\frac{G_{k}(s) K}{\tau_{d} s+1}\right\| \quad \text { and } \quad B_{2}:=h\left\|G_{k}(s) G_{k}(0)^{-1}+\frac{G_{k}(s) K s}{\tau_{d} s+1}\right\| .
$$

Define a normalized derivative action gain

$$
K=G_{k}(0)^{-1} K_{d n}
$$

We now investigate the optimal $K_{d n}$ which minimizes $B_{1}$ and $B_{2} / h$ in a combined fashion,

$$
B_{1}=\left\|\frac{F(s)-1}{s}+F(s) \frac{K_{d n}}{\tau_{d} s+1}\right\|, \quad \frac{B_{2}}{h}=\left\|F(s)\left(1+\frac{K_{d n}}{\tau_{d} s+1}\right)\right\|, \quad \text { where } \quad F(s)=G_{k}(s) G_{k}(0)^{-1},
$$

and $\tau_{d} \rightarrow 0$. Minimization of an expression in the form $B_{1}$ has been discussed in Reference 16. However, in this case we need to consider both $B_{1}$ and $B_{2}$ for

$$
F(s)=\frac{-102(s-1)}{(s+6)\left(s^{2}+8 s+17\right)}
$$

Figure 8 shows $B_{1}$ and $B_{2} / h$ values for different $K_{d n}$.

We observe that the minimum of $B_{1}$ occurs at $K_{d n}=0$; moreover the variance in $B_{1}$ is very small; on the other hand, $B_{2} / h$ varies significantly with $K_{d n}$. Considering that both $B_{1}$ and $B_{2}$ impacts $B$, a reasonable choice would be $K_{d n}=-0.23$, which leads to $B=1.653+h 1.034$. For $h=1 \mathrm{~s}$ we have $B^{-1}=0.3727$, which means that we can now choose $\left(\beta_{1}+\beta_{2}\right) \leq 0.3722$. The special choice $\beta_{1}=\beta_{2}=0.186$ leads to the PID controller (45)

$$
C_{P I D}(s)=-102\left(0.372+\frac{0.0346}{s}\right)\left(1-\frac{0.23 s}{\tau_{d} s+1}\right), \quad \tau_{d} \rightarrow 0
$$

With this controller, for $\tau_{d}=0$, the actual DM is $1.4757 \mathrm{~s}$, which means that compared with the PI controller the DM has been reduced (both designs satisfy minimum DM of $h=1 \mathrm{~s}$ ) but the step response with the PID controller is faster: settling time for $h=0$ has been reduced to $25 \mathrm{~s}$ from $35 \mathrm{~s}$.

Example 4. Let us now consider an infinite dimensional plant with three poles at $s=0$,

$$
P=\frac{0.8 e^{-0.5 s}(s-10)}{s^{3}(s+4)\left(s+e^{-s}\right)}
$$

In this case $G_{k}(0)=\left(s^{3} P\right)(0)=-2$. Accordingly, let $\tau_{d}=0$ and consider a PD-like controller

$$
Q(s)=\frac{-0.5\left(1+K_{d n} s\right)}{(1+\alpha s)^{2}} \quad \text { with } \quad \alpha=\frac{1}{20}
$$

Figure 9 shows $B_{1}$ and $B_{2} / h$ versus $K_{d n}$. In this case $B_{1}$ and $B_{2} / h$ are simultaneously minimized by $K_{d n}=-0.5646$, which gives $B_{1}=1.1046$ and $B_{2} / h=1$.

Now taking a desired minimum DM as $h=0.75 \mathrm{~s}$, we have $B^{-1}=1 / 1.8546=0.5392$. If we take $\beta_{1}=\beta_{2}=\beta_{3}=0.15$, then the condition (31) is satisfied with the resulting controller, (32),

$$
C(s)=C_{s}(s)=\frac{0.15\left(3 s^{2}+0.45 s+0.15^{2}\right)}{(1+0.05 \mathrm{~s})^{2}}(1-0.5646 \mathrm{~s}) .
$$




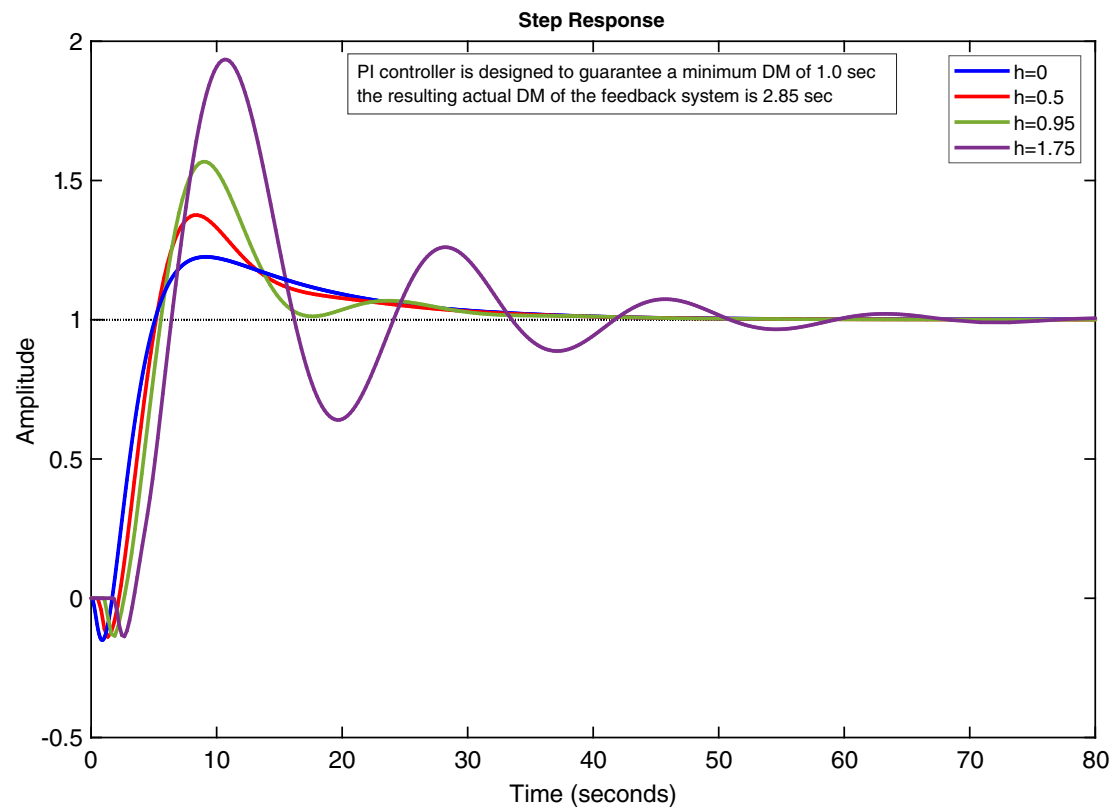

F I G U R E 7 Step response of the feedback system (PI controller for a fourth-order plant with one pole at $\mathrm{s}=0$ ) [Colour figure can be viewed at wileyonlinelibrary.com]

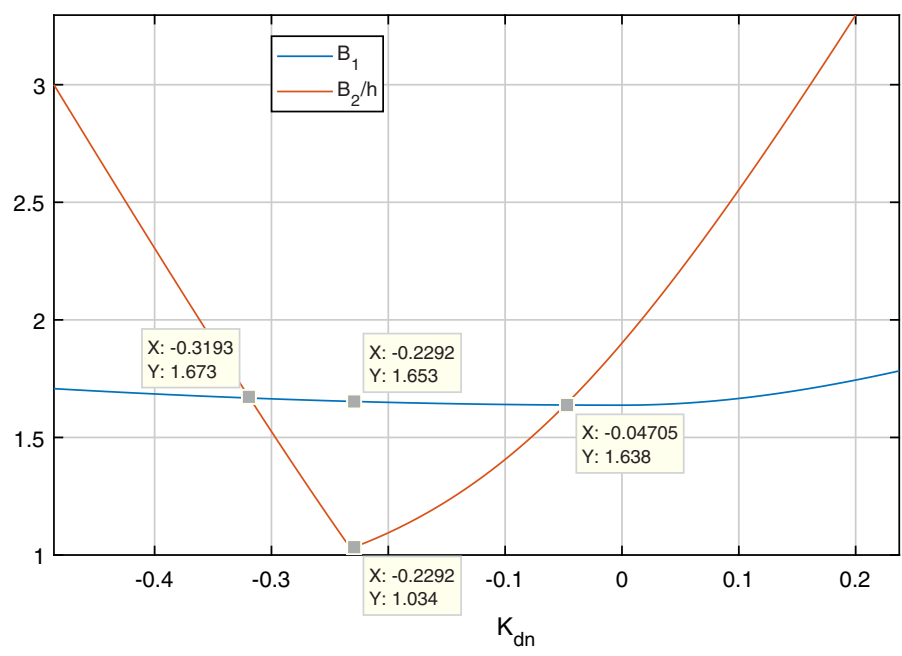

F I G U R E $8 \quad B_{1}$ and $B_{2} / h$ versus $K_{d n}$ [Colour figure can be viewed at wileyonlinelibrary.com]

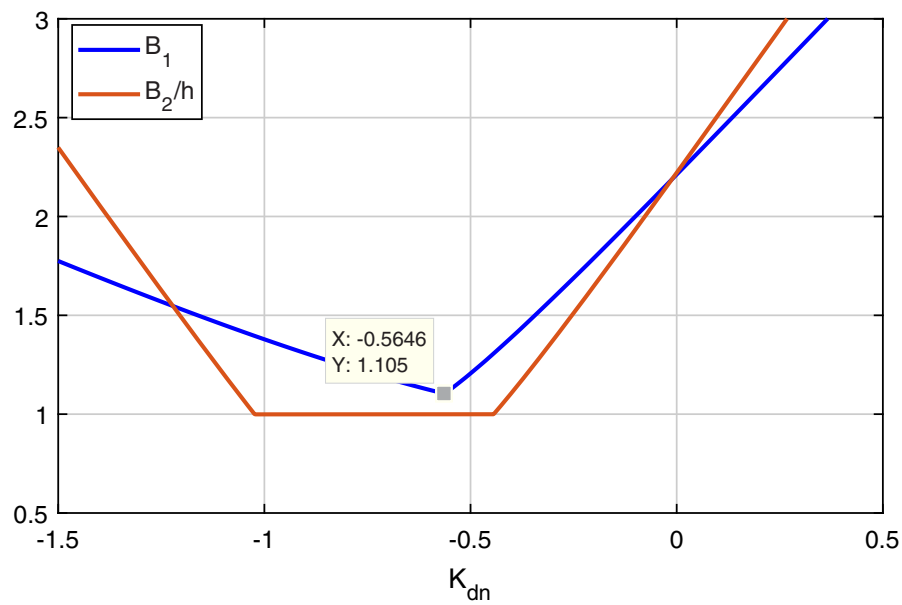

F I G U R E $9 \quad B_{1}$ and $B_{2} / h$ versus $K_{d n}$ [Colour figure can be viewed at wileyonlinelibrary.com] 
F I G U R E $10 \quad B_{1}$ and $B_{2} / h$ versus $K_{d n}$ [Colour figure can be viewed at wileyonlinelibrary.com]
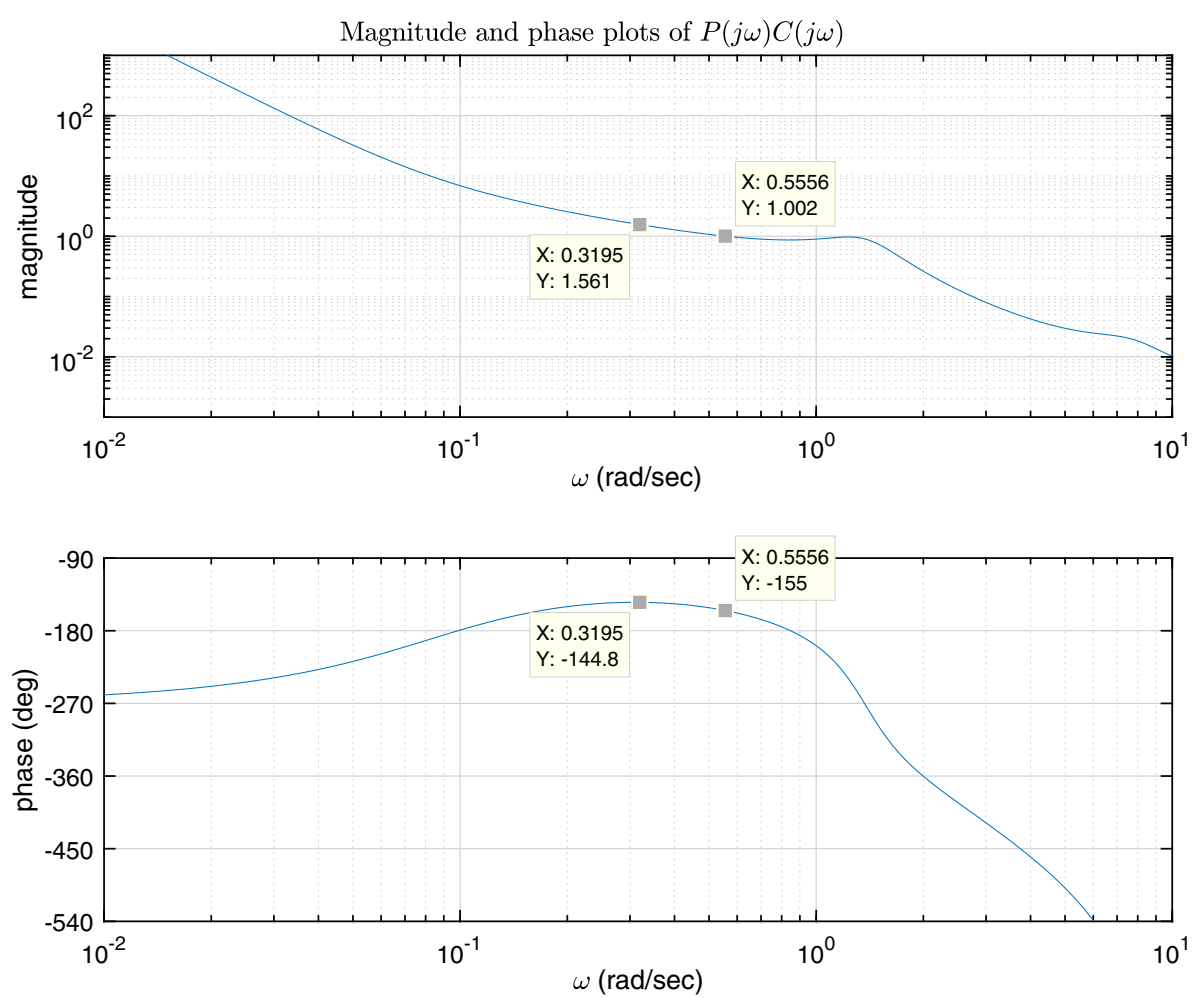

The Bode plots of $P C$ shown in Figure 10 illustrate that the system has phase margin of $25^{\circ}$ with crossover frequency $\omega_{c}=0.5575 \mathrm{rad} / \mathrm{s}$, which means that the actual delay margin is $0.7827 \mathrm{~s}$. Hence the objective of having at least $0.75 \mathrm{~s}$ DM is achieved. The closed-loop poles nearest to the Im-axis are at $-0.08 \pm j 0.9$, (computed with $\mathrm{QPmR}^{17}$ ). The Bode plots also show that if we modify the controller as $C=C_{S} C_{l a g}$ with the lag controller

$$
C_{\text {lag }}(s)=\frac{1+31.25 s}{1+1.56 \times 31.25 s}, \quad 0.032^{-1}=31.25
$$

then the crossover frequency moves to $0.324 \mathrm{rad} / \mathrm{s}$ and the phase margin increases to $33^{\circ}$, that gives an actual DM of $1.78 \mathrm{~s}$, which is far greater than the minimum required.

\section{6 | CONCLUSIONS}

In this article second-order unstable plants are considered first; we have proposed a PID controller design method which takes into account a lower bound of the delay margin and a pole placement condition. Then, arbitrary-order plants (can be infinite dimensional) with $k$ poles at $s=0$, and no other unstable poles, are studied. A low-order controller is designed to guarantee a desired level of delay margin, that gives a constraint on the pole placement. For systems with $k=1$ and $k=2$ it is possible to obtain PID controllers with this design even when the plant is infinite dimensional.

\section{ACKNOWLEDGMENT}

The authors would like to thank the Special Issue editors for exposing them to the problems studied here at earlier meetings and conferences.

\section{CONFLICT OF INTEREST}

The authors declared that they have no conflicts of interest to this work.

\section{ORCID}

Hitay Özbay (1D https://orcid.org/0000-0003-1134-0679 


\section{REFERENCES}

1. O’Dwyer A. Control of open-loop unstable processes with time delay using PI/PID controllers specified using tuning rules: an outline survey. Paper presented at: Proceedings of the 24th IET Irish Signals and Systems Conference; June 2013; Letterkenny, Ireland.

2. Vanavil B, Chaitanya KK, Rao AS. Improved PID controller design for unstable time delay processes based on direct synthesis method and maximum sensitivity. Int J Syst Sci. 2015;46(8):1349-1366.

3. Kishore CR, Sree RP. Tunning of PID controllers for unstable time delay systems using synthesis method. Paper presented at: Proceedings of the International Conference Intelligent Computing and Control System (ICICCS 2017); June 2017:765-770; Madurai, India.

4. Tan W, Marquez HJ, Chen T. IMC design for unstable processes with time delays. J Process Control. 2003;13:203-213.

5. Nikita S, Chidambaram M. Tuning of PID controllers for time delay unstable systems with two unstable poles. IFAC-PapersOnLine. 2016;49-1:801-806.

6. Ma D, Chen J, Liu A, Chen J, Niculescu SI. Explicit bounds for guaranteed stabilization by PID control of second-order unstable delay systems. Automatica. 2019;100:407-411.

7. Ma D, Chen J. Delay margin of low-order systems achievable by PID controllers. IEEE Trans Autom Control. 2019;64(5):1958-1973.

8. Qi T, Zhu J, Chen J. Fundamental limits on uncertain delays: when is a delay system stabilizable by LTI controllers? IEEE Trans Autom Control. 2017;62(3):1314-1328.

9. Özbay H, Gümüşsoy S, Kashima K, Yamamoto Y. Frequency domain techniques for $\mathrm{H}_{\infty}$ control of distributed parameter systems. Advances in Design and Control. Vol 32. Philadelphia PA: SIAM; 2018.

10. Gündeş AN, Özbay H. Controller redesign for delay margin improvement. Automatica. 2020;113:108790.

11. Niculescu S-I, Michiels W. Stabilizing a chain of integrators using multiple delays. IEEE Trans Autom Control. 2004;49(5):802-807.

12. Mendez-Barrios C, Michiels W, Niculescu SI. Further remarks on stabilizing chains of integrators by using network delays. Paper presented at: Proceeding of the 2009 European Control Conference; August 2009:383-388; Budapest, Hungary.

13. Ramirez A, Sipahi R. Multiple intentional delays can facilitate fast consensus and noise reduction in a multiagent system. IEEE Trans Cybern. 2019;49(4):1224-1235.

14. Ramirez A, Sipahi R. Single-delay and multiple-delay proportional-retarded (PR) protocols for fast consensus in a large-scale network. IEEE Trans Automat Contr. 2019;64(5):2142-2149.

15. Bedouhane F, Boussaada I, Niculescu S-I. Real spectral values coexistence and their effect on the stability of time-delay systems: Vandermonde matrices and exponential decay. Comptes Rendus Mathématique. 2020;358(9-10):1011-1032.

16. Özbay H, Gündeş AN. Resilient PI and PD controller designs for a class of unstable plants with I/O delays. Appl Comput Math. 2007;6(1):18-26.

17. Vyhlídal T, Zítek P. QPmR-quasi-polynomial root-finder: algorithm update and examples. In: Vyhlidal T, Lafay JF, Sipahi R, eds. Delay Systems. Advances in Delays and Dynamics. Vol 1. Cham, Switzerland: Springer; 2014:299-312.

How to cite this article: Özbay H, Gündes AN. PID and low-order controller design for guaranteed delay margin and pole placement. Int J Robust Nonlinear Control. 2021;1-14. https://doi.org/10.1002/rnc.5521 